Progress in Nuclear Science and Technology

Volume 6 (2019) pp. 113-116

\title{
ARTICLE
}

\section{Measurement of radiation dose distribution in minipig physical phantom using RPLGD}

\author{
Jae Seok Kimª, Pilsoo Lee ${ }^{\mathrm{a}}$, Byeong Ryong Park ${ }^{\mathrm{a}}$, Han Sung Kimª, Kum Bae Kim ${ }^{\mathrm{b}}$, Gyu Seok Cho ${ }^{\mathrm{b}}$, Sehwan Shim ${ }^{\mathrm{b}}$, \\ Wi-Ho Ha ${ }^{a^{*}}$, Sunhoo Park ${ }^{\mathrm{a}}$ and Young-Woo Jin ${ }^{\mathrm{a}}$ \\ ${ }^{a}$ National Radiation Emergency Medical Center, Korea Institute of Radiological and Medical Sciences, 75, Nowon-ro, Nowon-gu, \\ Seoul, Republic of Korea ${ }^{b}$ Korea Cancer Center Hospital, Korea Institute of Radiological and Medical Sciences, 75, Nowon-ro, \\ Nowon-gu, Seoul, Republic of Korea
}

\begin{abstract}
Radio-photoluminescence glass dosimeter (RPLGD) has many advantages such as small size, high sensitivity, low fading effect, excellent dose linearity and repeatability. Because of these advantages, RPLGDs were used to measure the absorbed dose as in-vivo dosimeter. In Korea Institute of Radiological and Medical Sciences (KIRAMS), a minipig physical phantom was applied to evaluate the dose distribution for animal irradiation study. In this study, RPLGDs were used as a dosimeter to evaluate the dose distribution in the $5^{\text {th }}$ and $25^{\text {th }}$ slices. For the reliable result, RPLGDs were irradiated to evaluate the expanded measurement uncertainty. The expanded measurement uncertainty of RPLGD was determined as $6.08 \%$ with a coverage factor of $k=2$. Additionally, the framework of the Geant4 toolkit was used to verify the dose distribution by using RPLGD measurement under the same experiment condition. To analyze the linearity between the RPLGD measurements and Geant 4 simulation, each of absorbed doses was compared according to the measurement points in the minipig physical phantom. As a result, the coefficients of determination $\left(\mathrm{R}^{2}\right)$ were evaluated as 0.9767 and 0.9852 at each slice of the $5^{\text {th }}$ and $25^{\text {th }}$.
\end{abstract}

Keywords: RPLGD; Geant4 toolkit; minipig physical phantom; absorbed dose; dose distribution

\section{Introduction}

In an incident of excessive radiation exposure, evaluation of exposed dose is important to decide the subsequent therapy method. In general, human phantoms composed of major organs and tissues can be used to evaluate the organ dose. However, a study on the direct effect of radiation exposure is difficult to carry out in human body. In KIRAMS, a minipig was chosen as a new animal model because of its similarities in anatomy and pathology to human body [1]. In the present study, the minipig physical phantom was applied to evaluate the dose distribution for animal irradiation study. To evaluate the dose distribution in the minipig physical phantom, RPLGD was used as an in-vivo dosimeter. Many types of radiation detector had been developed such as metal-oxide-semiconductor field effect transistor (MOSFET), thermoluminescence dosimeter (TLD) and optically stimulated luminescence dosimeter (OSLD). MOSFET can measure a signal in the real-time and excellent reproducibility of signal, but it has dependence of direction and energy [2]. TLD has smaller effective volumes than ion-chamber, but it cannot repeat the readout and it has high energy and direction dependency due to comparative high uncertainty in the range of $\mathrm{X}$-ray energy. OSLD presents not only the advantages of

*Corresponding author. Email: lovin@kirams.re.kr analyzing quick measurements but also minimal signal loss for repeated readout signal. However, it has disadvantage of accumulating the residual signal in deeper energy traps $[3,4]$. RPLGD has been widely used to measure the absorbed dose as the in-vivo dosimeter. In comparison with other radiation dosimeters, RPLGDs have many advantages such as small size, high sensitivity, low fading effects, excellent dose linearity, and repeatability. Because of these reasons, the RPLGDs have been used as in-vivo dosimetry in various fields for observing the exposed dose [5]. In this study, the expanded measurement uncertainty of RPLGD was evaluated at irradiation dose of $1 \mathrm{~Gy}$, and the RPLGDs were used to evaluate the dose distribution in the minipig physical phantom. In addition, Monte-Carlo simulations with a computational minipig phantom were performed to evaluate the dose distribution under the same experiment condition using the Geant 4 toolkit.

\section{Methods}

\subsection{Minipig physical phantom}

To evaluate the dose distribution in animal irradiation study, the minipig physical phantom was developed based on computed tomography (CT) data. The minipig physical phantom has three major organs, bone, lung, 
and intestine, separately, and the rest parts were simply regarded as a soft tissue. The major organs of minipig physical phantom were distinguished by color according to different materials. In the present phantom, materials of phantom were fabricated by tissue equivalent (TE) substitutes. The soft tissue was composed of acrylonitrile butadiene styrene (ABS), which has a similar mass density in comparison with human soft tissue. Fiber-reinforced plastic (FPR) and Polycoat CH-304 (Aekyung, Korea) were chosen as a base of bone material. Non-foaming urethane was chosen to be a lung material as a TE substitute. Table 1 shows the density and substitute materials used in the minipig physical phantom. Figure 1(a) shows that the minipig physical phantom was sliced into 27 slices each of which has dosimeter holes in a grid pattern with an interval of $15 \mathrm{~mm}$, except for head and tail parts. Figures 1(b) and 1(c) show the cross section of the $5^{\text {th }}$ and $25^{\text {th }}$ slices in the minipig physical phantom.

Table 1. Density and substitute materials of the minipig physical phantom.

\begin{tabular}{ccc}
\hline $\begin{array}{c}\text { Type of } \\
\text { organs/tissue }\end{array}$ & $\begin{array}{c}\text { Density } \\
\left(\mathrm{g} / \mathrm{cm}^{3}\right)\end{array}$ & Substitute materials \\
\hline Soft tissue & 1.0 & ABS \\
Bone & 1.4 & FPR and Polycoat CH-304 \\
Lung & 0.5 & Non-foaming urethane \\
\hline
\end{tabular}

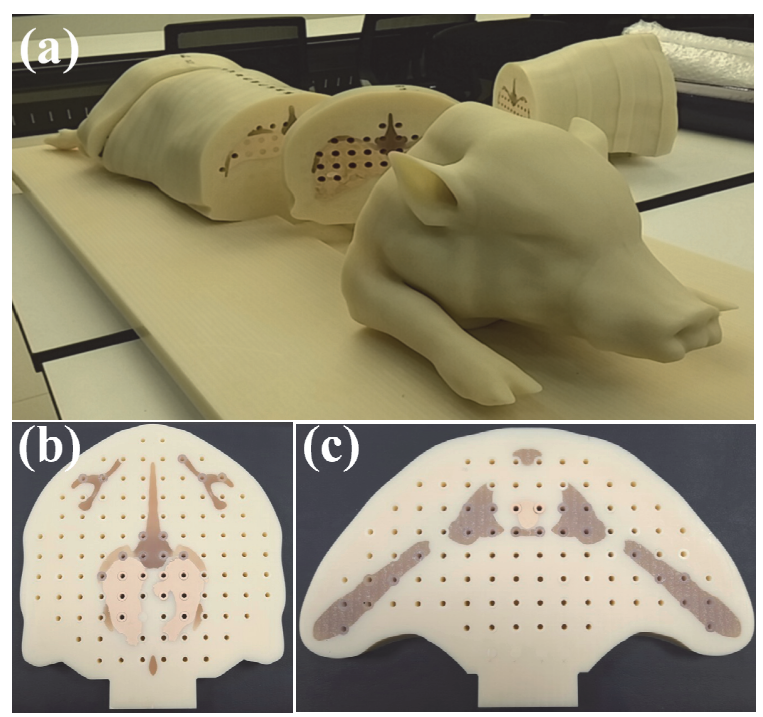

Figure 1. Geometry of the minipig physical phantom: (a) the minipig physical phantom sliced into 27 slices, cross section of the (b) $5^{\text {th }}$ and (c) $25^{\text {th }}$ slices.

\subsection{RPLGD}

To evaluate the dose distribution, a model GD-302M RPLGD (AGC Techno Glass Corporation, Japan) and FGD-1000 automatic reader were used. The geometry of GD-302M is $1.5 \mathrm{~mm}$ in diameter and $12 \mathrm{~mm}$ in length. The weight composition of RPLGD was as follows: $51.2 \% \mathrm{O}, 11.0 \% \mathrm{Na}, 6.1 \% \mathrm{Al}, 31.5 \% \mathrm{P}$, and $0.2 \% \mathrm{Ag}$, with a mass density of $2.61 \mathrm{~g} / \mathrm{cm}^{3}$ [6]. These materials composed of the silver-activated phosphate glass in RPLGD. When ionizing radiation irradiated the silver-activated phosphate glass, stable radio-photo luminescence (RPL) centers appeared in RPLGD. When RPLGD was excited by an ultraviolet (UV) laser, orange luminescence would be generated from RPL center [7]. RPLGDs can be read out using two types of magazine having structural difference, which allow RPLGD to read a wide range of absorbed dose. Each of magazines can be used to read out the dose ranges from $10 \mu \mathrm{Gy}$ to $10 \mathrm{~Gy}$ and from $1 \mathrm{~Gy}$ to $500 \mathrm{~Gy}$ [8]. In order to use RPLGDs as a dosimeter, dose response curve was established by evaluating for the linear relationship. RPLGDs were irradiated by using the ${ }^{60} \mathrm{Co}$ teletherapy unit (Best theratronics, Canada), which has a delivered dose rate of $183.06 \mathrm{cGy} / \mathrm{min}$ at a source to surface distance (SSD) of $80 \mathrm{~cm} .{ }^{60} \mathrm{Co}$ source has a half-life of 5.271 years and emits gamma-rays having energies of 1.173 and $1.332 \mathrm{MeV}$. Furthermore, to progress in a quantitative experiment, the solid water phantom was manufactured to read out the absorbed dose at a depth of $5 \mathrm{~mm}$. Solid water phantom is made with the polymethyl methacrylate (PMMA, $1.19 \mathrm{~g} / \mathrm{cm}^{3}$ ), which is commonly used as TE substitute. Meanwhile, to evaluate the measurement uncertainty, 40 different RPLGDs were irradiated to $1 \mathrm{~Gy}$ at a depth of $5 \mathrm{~mm}$ under solid water phantom.

\subsection{Experimental setup}

For a dosimetry purpose, RPLGDs were used to measure the dose distribution in the minipig physical phantom. Figure 2(a) shows the experimental setup for array of irradiating gamma-ray at the $5^{\text {th }}$ slice. The gamma-rays were provided by a ${ }^{192} \mathrm{Ir}$ source, which has a half-life of 78.827 days and emits complicated gamma-rays spectrum from 136.4 to $884.5 \mathrm{keV}$. The activity of ${ }^{192} \mathrm{Ir}$ source used in this study is about 2.006 Ci. The irradiation area of ${ }^{192} \mathrm{Ir}$ source is $5 \times 5 \mathrm{~cm}^{2}$, which was produced using seven-catheters at interval of $2.5 \mathrm{~mm}$ on the bolus (Radiation Product Design, Inc., USA) with a thickness of $10 \mathrm{~mm} .{ }^{192} \mathrm{Ir}$ source moved along with a catheter at an interval of $2.5 \mathrm{~mm}$. The bolus was used for irradiating uniformly on the surface of the minipig physical phantom. The bolus has a TE density of approximately $1.03 \mathrm{~g} / \mathrm{cm}^{3}$. Using the high-dose-rate (HDR) brachytherapy afterloader (Varian Medical System, USA) with radiation treatment planning (RTP), irradiation field was determined to reflect the same condition as a local radiation exposure in this study. Figure 2(b) shows the experimental setup for measuring dose distribution using RPLGDs, which were inserted in holders. Each of the $5^{\text {th }}$ and $25^{\text {th }}$ slices was irradiated by 10 and 20 Gy under the same experiment condition. In addition, using the Geant 4 toolkit, the computational minipig phantom based on the voxel model was used for verifying the dose distribution in the minipig physical phantom. 


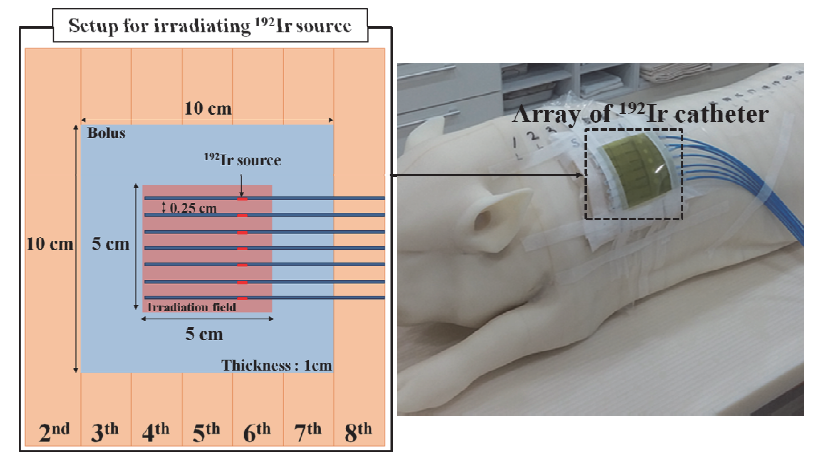

(a)

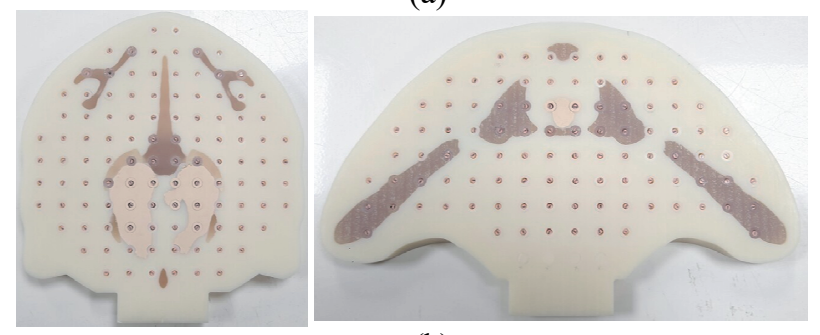

(b)

Figure 2. Experimental setup for measuring the dose distribution: (a) ${ }^{192} \mathrm{Ir}$ catheter setup for irradiating at the $5^{\text {th }}$ slice, (b) RPLGDs inserted in the $5^{\text {th }}$ and $25^{\text {th }}$ slices of the minipig physical phantom.

\section{Results and discussion}

\subsection{Measurement results of RPLGD}

Figure 3 shows the dose linearity between measured values of RPLGD and irradiation doses at a depth of 5 mm under solid water phantom. Experimental setup was designed according to IAEA TRS-398 protocol for determination of irradiation dose. The measurements were carried out using a water phantom, and a Farmer type ionization chamber (EXRADIN A12, Standard Imaging, USA) connected to a SuperMAX electrometer (Standard Imaging, USA).

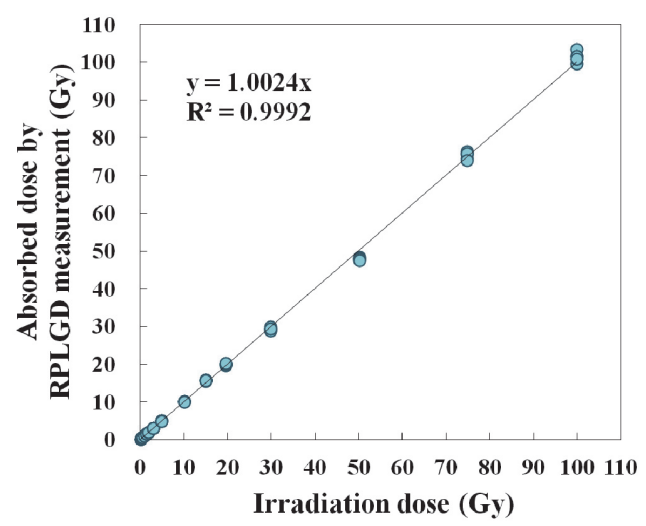

Figure 3. Linearity between measurement and irradiation doses at a depth of $5 \mathrm{~mm}$ in the solid water phantom.

This result was found that RPLGDs have a linear relationship for absorbed doses ranging from 0.1 to 100 Gy with the $\mathrm{R}^{2}$ of 0.9992 . Each of absorbed doses was evaluated by 6 RPLGDs at one irradiation dose, which ranged from 0.1 to $100 \mathrm{~Gy}$. In this regard, dose response curves were used for evaluating the absorbed dose.

Figure 4 shows absorbed doses measured from 40 different RPLGDs. As a result, we found two types of standard deviation from repeated measurements using one RPLGD in the FGD-1000 automatic reader and single measurement using different RPLGDs. The expanded uncertainty of RPLGD measurement was determined as $6.08 \%$ with a coverage factor of $k=2$. Therefore, this result demonstrated that even if RPLGD was chosen randomly to evaluate the absorbed dose, absorbed dose by RPLGD measurement can be valid within a confidence level of $95 \%$.

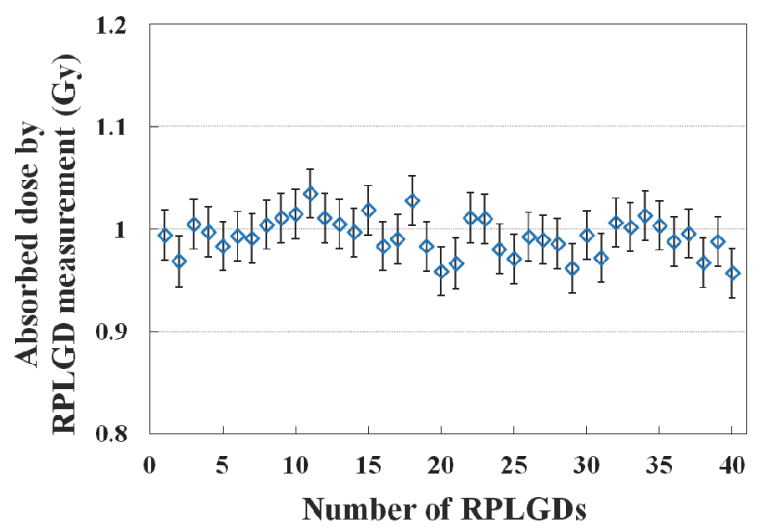

Figure 4. Absorbed dose by measurements of 40 different RPLGDs at $1 \mathrm{~Gy}$.

\subsection{Linearity between measurement and simulation}

To evaluate the dose distribution in the minipig physical phantom at the $5^{\text {th }}$ and $25^{\text {th }}$ slices, most of holes in the slices were used for the measurement points. RPLGDs were inserted in holes of the slices, which have the number of 104 and 96 holes. The measured counts of RPLGDs were converted to absorbed dose at measurement points. In addition, Geant4 toolkit was used to calculate absorbed dose under the same experiment condition. In order to analyze the linearity between measurement and calculation, each of absorbed doses was evaluated. Figure 5 shows the linearity of absorbed doses between RPLGD measurements and Geant4 simulation according to position of RPLGD in the $5^{\text {th }}$ and $25^{\text {th }}$ slices. The values of $\mathrm{R}^{2}$ were evaluated as 0.9767 and 0.9852 . $\mathrm{R}^{2}$ could explain the linearity of absorbed doses according to regression curve. As a result, $\mathrm{R}^{2}$ indicated the good linearity relationship of dose distribution between RPLGD measurements and Geant 4 simulation at the $5^{\text {th }}$ and $25^{\text {th }}$ slices. From the results of this comparison between experiment and simulation, we conclude that RPLGDs can be used effectively to evaluate the dose distribution in the minipig physical phantom. 


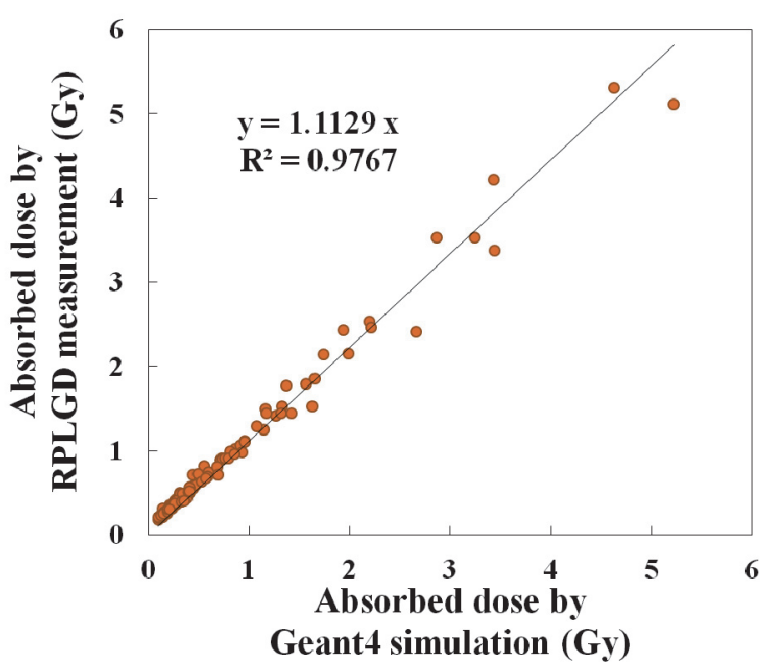

(a)

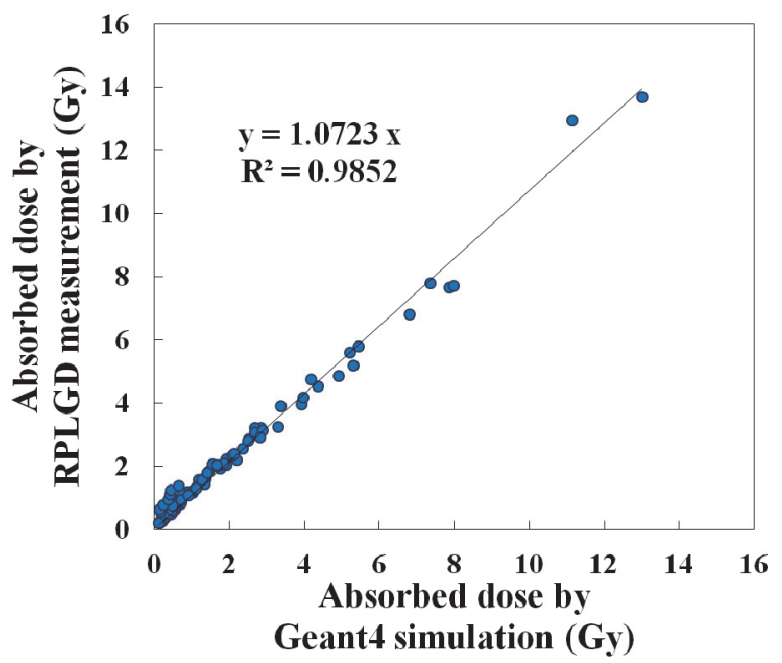

(b)

Figure 5. Linearity of absorbed doses between RPLGD measurement and Geant 4 simulation: (a) the $5^{\text {th }}$ slice and (b) $25^{\text {th }}$ slice.

\section{Conclusion}

RPLGD has many advantages such as small size, high sensitivity, low fading effect, excellent dose linearity, and repeatability. These advantages as a dosimeter enable to evaluate the dose distribution in the minipig physical phantom. Expanded measurement uncertainty of RPLGDs was evaluated as $6.08 \%$ at irradiation dose of $1 \mathrm{~Gy}$. In order to verify the dose distribution, Geant4 toolkit was used to calculate the absorbed dose at the same measurement points along with dosimeter position. As a result of linearity according to regression curve, the values of $\mathrm{R}^{2}$ were evaluated as 0.9767 and 0.9852 at the $5^{\text {th }}$ and $25^{\text {th }}$ slices, respectively. Consequently, RPLGDs can be expected to be used as in-vivo dosimeter. For future investigation, we would evaluate not only dose distribution, but also the correction factors for accurate dose assessment. Therefore, it would be necessary to develop methodology for evaluating the accurate absorbed dose with RPLGDs, which require various correction factors according to irradiation condition.

\section{Acknowledgements}

This study was supported by a grant of the Korea Institute of Radiological and Medical Sciences (KIRAMS), funded by Ministry of Science, ICT and Future Planning, Republic of Korea (1711045573;1711045551/50586-2017).

\section{References}

[1] J-G. Lee, S. Park, C-H. Bae, W.-S. Jang, S-J. Lee, D.N. Lee, J.K. Myung, C.H. Kim, Y-W. Jin, S-S. Lee and S. Shim, Development of a minipig model for lung injury induced by a single high-dose radiation exposure and evaluation with thoracic computed tomography, J. Radiat. Res. 57 (2016), pp. 201-209.

[2] J-E. Rah, D.H. Oh, D. Shin, S.B. Lee, T.H. Kim, J-Y. Kim, Y. Kase, Z. Li, G.S. Ibbott, P.J. Koss, L. Lin, J. McDonough, B. Arjomandy and S.Y. Park, Feasibility study of glass dosimeter for postal dose intercomparison of high-energy proton therapy beams, Radiat. Meas. 59 (2013), pp. 66-72.

[3] K. Son, H. Jung, S.H. Shin, H-H. Lee, M-S. Kim, Y.H. Ji and K.B. Kim, Evaluation of the dosimetric characteristics of a radiophotoluminescent glass dosimeter for high-energy photon and electron beams in the field of radiotherapy, Radiat. Meas. 46 (2011), pp. 1117-1122

[4] J-E. Rah, J-Y. Hong, G-Y. Kim, Y-L. Kim, D-O. Shin and T-S. Suh, A comparison of the dosimetric characteristics of a glass rod dosimeter and a thermoluminescent dosimeter for mailed dosimeter, Radiat. Meas. 44 (2009), pp. 18-22.

[5] H. Yamazaki, K. Iwama, T. Nishimura, Y. Iwai, N. Aibe, T. Takenaka, S. Miyake, E. Tanaka, K. Yoshida, Y. Oota, H. Ikeno, S. Nakamura and H. Okabe, Comparison of calculated dose by helical tomotherapy treatment planning machine and measured dose of radiophotoluminescence glass dosimeter in lung lesions using rando phantom, Anticancer Res. 33 (2013), pp. 1679-1684.

[6] D.Y. Huang and S-M. Hsu, Radio-photoluminescence glass dosimeter (RPLGD), Advances in Cancer Therapy, H. Gali-Muhtasib, InTech, Ch.25, 553-568 (2011), ISBN 9789533071.

[7] J-E. Rah, D.H. Oh, D. Shin, D-H. Kim, Y.H. Ji, J.W. Kim and S.Y. Park, Dosimetric evaluation of a glass dosimeter for proton beam measurements, Appl. Radiat. Isot. 80 (2012), pp. 1616-1623.

[8] H. Mizuno, T. Kanai, Y. Kusano, S. Ko, M. Ono, A. Fukumura, K. Abe, K. Nishizawa, M. Shimbo, S. Sakata, S. Ishikura and H. Ikeda, Feasibility study of glass dosimeter postal dosimetry audit of high-energy radiotherapy photon beams, Radiother. Oncol. 86 (2008), pp. 258-263. 